\title{
Search of antimicrobial lactic acid bacteria from Salmonella-negative dogs
}

Estrella Jimenez-Trigos ${ }^{1 *}$, Marion Toquet ${ }^{1}$, Marta Barba , Ángel Gómez-Martín' ${ }^{1}$ Juan J. Quereda² and

Esther Bataller ${ }^{1^{*}}$

\begin{abstract}
Background: Salmonellosis is one of the most important food-borne zoonotic disease affecting both animals and humans. The objective of the present study was to identify gastrointestinal (GI) lactic acid bacteria (LAB) of canineorigin from Salmonella-negative dogs' faeces able to inhibit monophasic Salmonella Typhimurium previously isolated from dogs' faeces, in order to be used as a potential probiotic in pet nutrition.

Results: Accordingly, 37 LAB were isolated from Salmonella-negative dogs' faeces and tested against monophasic $S$. Typhimurium using the spot on lawn method out of which 7 strains showed an inhibition halo higher than $2.5 \mathrm{~cm}$. These 7 strains were also tested with the co-culture method and one showed the greatest inhibition value $(p<0.05)$. Subsequently, the isolate was identified through $16 \mathrm{~S}$ rRNA sequencing and sequence homology and designated as Ligilactobacillus salivarius (L. salivarius). LAB from Salmonella-positive dogs were also identified and none was the selected strain. Finally, to identify the mechanism of inhibition of L. salivarius, the supernatant was analyzed, and a dose response effect was observed.
\end{abstract}

Conclusions: It is concluded that the canine-origin L. salivarius, could possess some in vitro functional attributes of a candidate probiotic and could prevent monophasic $S$. Typhimurium colonization or inhibit its activity if the infection occurs.

Keywords: Lactic acid bacteria, Salmonella, Probiotics, Ligilactobacillus salivarius, Lactobacillus, Dogs

\section{Background}

Antibiotics have been widely used in small animals' veterinary practices for treatment of some medical conditions. However, overuse of antimicrobials in pets happens and during the last decades we have witnessed how the indiscriminate use of antibiotics has resulted in the emergence of multidrug-resistant strains even in pets [1-5]. It is estimated that over $70 \%$ of the bacteria responsible

\footnotetext{
*Correspondence: estrella.jimenez@uchceu.es; esther.bataller@uchceu.es ${ }^{1}$ Microbiological Agents Associated with Animal Reproduction (ProVaginBIO) Research Group, Departamento Producción y Sanidad Animal, Salud Pública Veterinaria y Ciencia y Tecnología de los Alimentos, Facultad de Veterinaria, Universidad Cardenal Herrera-CEU, CEU Universities, Carrer Tirant lo Blanc, 7, 46115 Alfara del Patriarca, Valencia, Spain

Full list of author information is available at the end of the article
}

for healthcare associated infections are resistant to at least one of the antibiotics used worldwide as a first-line therapy [6, 7]. Antimicrobial resistance (AMR) is a global threat worldwide. According to the tenth ESVAC report (European Monitoring of Veterinary Antimicrobial Consumption), the consumption of antimicrobials in Spain is one of the highest among European countries [8].

Antibiotic resistance in enteric pathogens such as Salmonella spp. is a major concern for public health safety. According to The European Food Safety Authority [9], salmonellosis is the second most commonly reported gastrointestinal infection in humans and in 2019, 87,923 confirmed cases were reported, many of which are due to antibiotic-resistant Salmonella spp.

Several studies have been conducted worldwide to assess the prevalence of Salmonella spp. in clinically 
healthy and diarrheic dogs [10-14]. However, prevalence in clinically healthy dogs varies notably and may even be different depending on the country [15]. It has been shown that healthy dogs can harbour Salmonella spp. and eating contaminated foods, including unprocessed or raw dog food, especially raw meat, has been related as one of the most important risk factors of Salmonella spp. carriage [10, 13]. Several authors have reported antibiotic resistance in Salmonella isolates from dogs [10, 11, 15, $16]$ and, as they are often in close contact with humans, they can be a source or a recipient of resistant bacteria such as Salmonella spp. [17]. Faced with this complex situation, the Spanish National Plan Against Antibiotic Resistance (PRAN) was approved in 2014 with the objective of reducing the risk of dissemination of resistance to antibiotics and has provided numerous achievements, including the decrease of $7.2 \%$ of antibiotics consumption in human health, and $14 \%$ in veterinary medicine. However, the lack of alternative treatments could be one of the biggest problems of public health.

A recent study made in Spain showed a relationship between LAB content and the absence of Salmonella spp. in dogs' faeces [14]. LAB are the microorganisms most commonly used as probiotics which can be used to prevent infections or as alternatives of antibiotics. Certain probiotic strains of Lactobacillus have been reported to be effective against microbial Gram-negative pathogens involved in diarrhoea, gastroenteritis, urovaginal infections and inflammatory bowel disease $[18,19]$ and also can exert a beneficial effect on the intestinal microbiota increasing the number of lactobacilli and modulating the physiology and immunity parameters of dogs microbiota [20]. Several studies have reported a reduction of Salmonella spp. in both chicken and poults after using probiotics $[21,22]$. However, studies using probiotics of canine origin, from different dogs breeds, are very limited and this has become of great interest in the scientific canine community [23]. To the best of our knowledge, no studies have demonstrated if GI LAB from Salmonella-negative dogs are able to inhibit this pathogen and consequently protect them against the infection.

In that sense, since a key tool to cope with microbial resistance is prevention, the main objective of our study was to identify antimicrobial LAB from Salmonella-negative dogs able to inhibit monophasic $S$. Typhimurium isolated from Salmonella-positive dogs. Secondly, this study aimed to find out if the LAB identified in Salmonella-negative dogs were isolated from Salmonellapositive dogs. Finally, the purpose of this study was to explain if GI LAB with antimicrobial activity from Salmonella-negative dogs could justify the absence of the pathogen or could protect dogs against monophasic $S$. Typhimurium carriage.

\section{Results}

Obtention of LAB isolates

Thirty-seven GI LAB were randomly selected from different Salmonella-negative dogs isolated and grown in Man, Rogosa and Sharpe (MRS) agar plates as it was described in a previous study [14]. LAB were obtained from males and females of different breeds and ages, housed in different environments.

\section{Inhibition assays in solid and liquid media: spot-on- lawn and co-culture methods Inhibition assays in solid media: spot-on-law method}

Results obtained from the spot-on-lawn technique indicated that the median value of the diameter of the inhibition halos was significantly higher than $1.5 \mathrm{~cm}(p<0.01)$. Several LAB were able to inhibit monophasic $S$. Typhimurium with a diameter of inhibition higher than $2.0 \mathrm{~cm}$ (Table 1). The greatest inhibition values corresponded to isolates $8,12,15,18,19,30,36$. The halo diameter of seven $\mathrm{LAB}$ chosen for the next co-culture assay were significantly higher than $2.5 \mathrm{~cm}(p<0.05)$. The antibiotic (ciprofloxacin) used as control showed an average inhibition halo of $2.04 \mathrm{~cm}$ (Fig. 1).

\section{Inhibition assays in liquid media: co-culture method}

In the co-culture method, two parameters were evaluated with these seven selected BAL from the spot-on-lawn method. Firstly, we studied in Xylose Lysine Deoxycholate (XLD) agar the inhibition of monophasic $S$. Typhimurium by LAB compared with the control $L$. reuteri Protectis $^{\circledR}$ (Fig. 2).

Table 1 Average and standar desviation inhibition halo $(\mathrm{cm})$ of the thirty-seven $L A B$ in the spot-on lawn assay

\begin{tabular}{llllll}
\hline LAB & $\begin{array}{l}\text { Average } \\
\text { inhibition halo } \\
(\mathbf{c m})\end{array}$ & & LAB & $\begin{array}{l}\text { Average } \\
\text { inhibition halo } \\
(\mathbf{c m})\end{array}$ & $\begin{array}{l}\text { LAB } \\
\text { Average } \\
\text { inhibition } \\
\text { halo }(\mathbf{c m})\end{array}$ \\
\hline 1 & $1.800 \pm 0.265$ & 13 & $2.200 \pm 0.100$ & 25 & $1.667 \pm 0.577$ \\
2 & $0.900 \pm 0.100$ & 14 & $2.033 \pm 0.058$ & 26 & $1.600 \pm 0.600$ \\
3 & $1.600 \pm 0.265$ & 15 & $\mathbf{3 . 0 0 0} \pm \mathbf{0 . 1 0 0}$ & 27 & $2.000 \pm 0.436$ \\
4 & $1.667 \pm 0.289$ & 16 & $1.933 \pm 0.208$ & 28 & $1.967 \pm 0.153$ \\
5 & $2.367 \pm 0.252$ & 17 & $2.200 \pm 0.500$ & 29 & $2.000 \pm 0.100$ \\
6 & $1.800 \pm 0.346$ & 18 & $\mathbf{2 . 5 3 3} \pm \mathbf{0 . 4 5 1}$ & 30 & $\mathbf{3 . 0 0 0} \pm \mathbf{0 . 1 0 0}$ \\
7 & $1.800 \pm 0.361$ & 19 & $\mathbf{2 . 9 0 0 \pm 0 . 2 0 0}$ & 31 & $2.400 \pm 0.458$ \\
8 & $\mathbf{2 . 8 5 0} \pm \mathbf{0 . 5 6 8}$ & 20 & $2.433 \pm 0.058$ & 32 & $1.933 \pm 0.503$ \\
9 & $2.200 \pm 0.200$ & 21 & $2.067 \pm 0.306$ & 33 & $2.100 \pm 0.100$ \\
10 & $1.967 \pm 1.060$ & 22 & $2.100 \pm 0.265$ & 34 & $2.367 \pm 0.551$ \\
11 & $0.000 \pm 0.000$ & 23 & $2.267 \pm 0.551$ & 35 & $0.000 \pm 0.000$ \\
12 & $\mathbf{2 . 6 0 0} \pm \mathbf{0 . 1 0 0}$ & 24 & $1.700 \pm 0.265$ & 36 & $\mathbf{3 . 1 0 0} \pm \mathbf{0 . 1 7 3}$ \\
& & & & 37 & $1.533 \pm 0.321$ \\
\hline
\end{tabular}




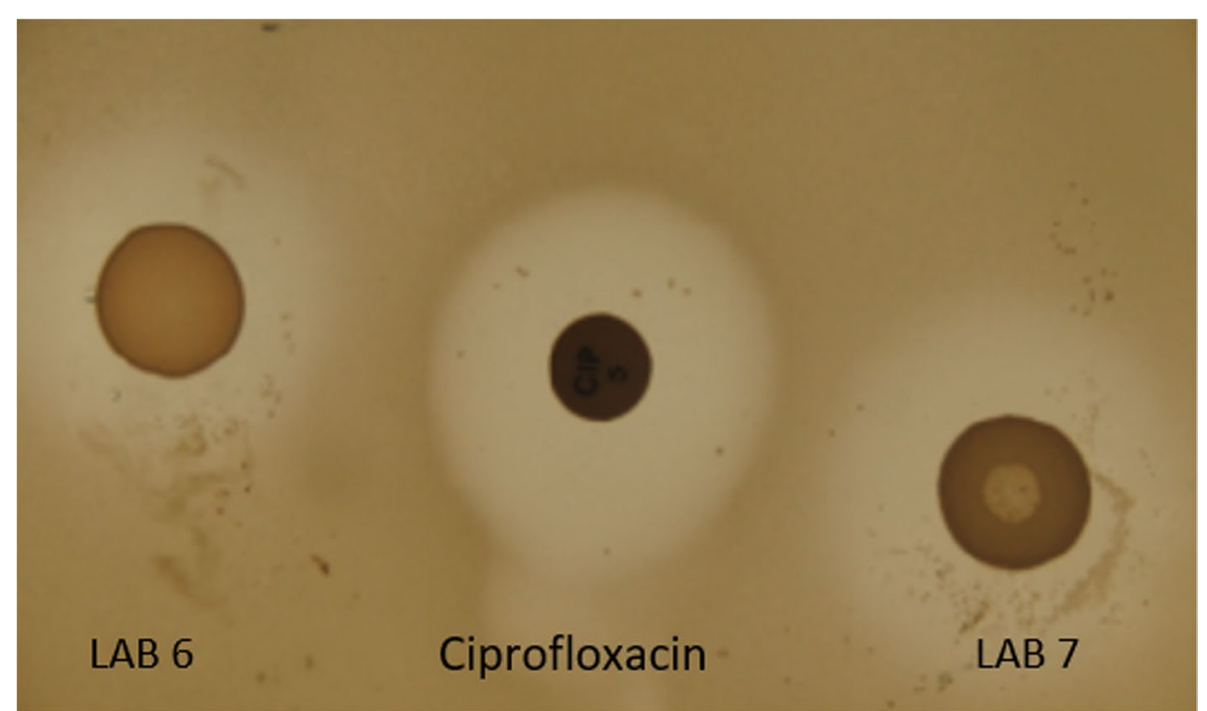

Fig. 1 Inhibition halos of $L A B$ 6, 7 and the antibiotic ciprofloxacin with the spot-on-lawn method

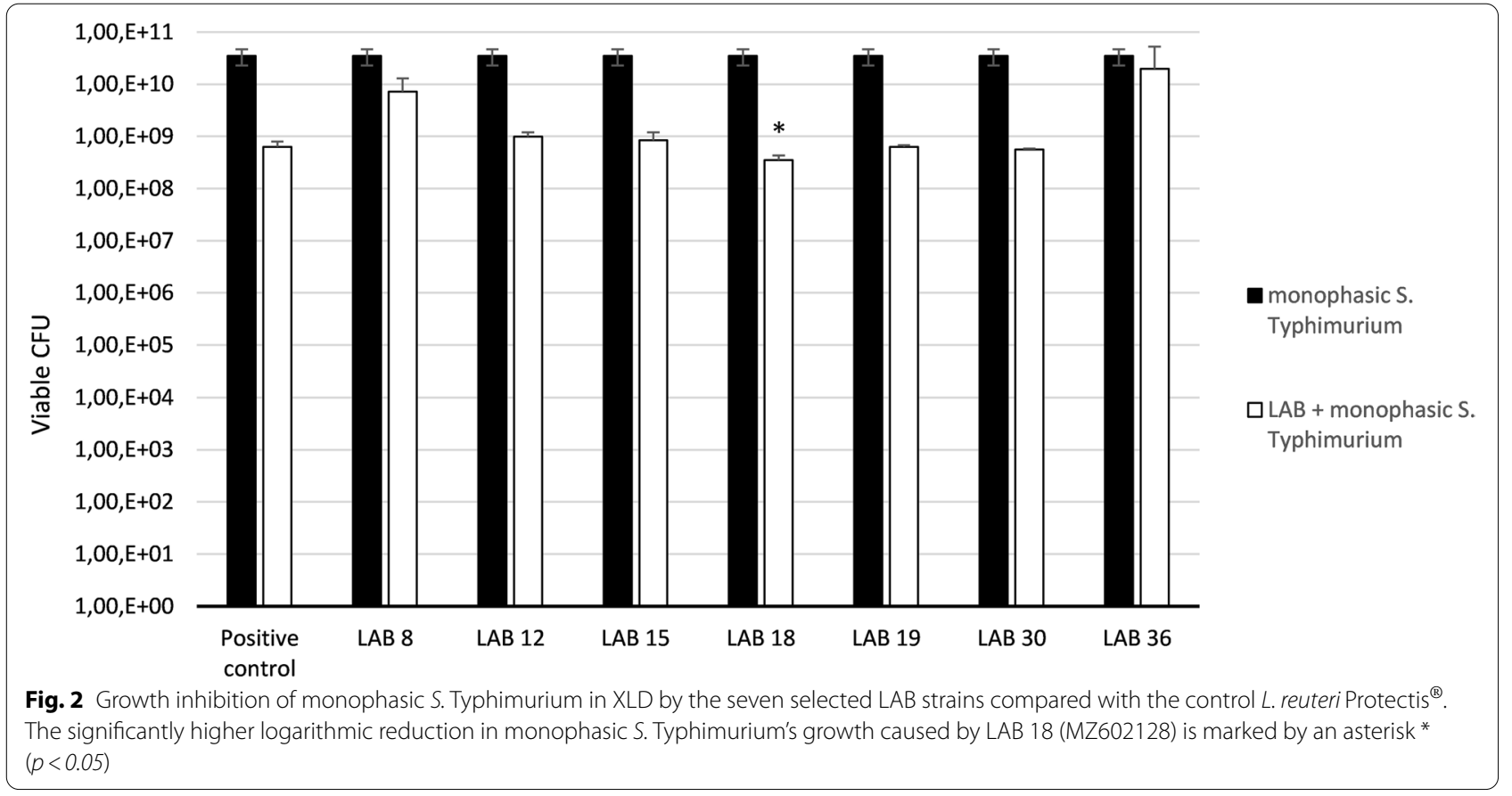

On the other hand, we studied the survival of these LAB after incubation with monophasic S. Typhimurium using MRS plate agar (Fig. 3).

Results of the inhibition assay in XLD medium and in MRS medium indicated that LAB 18, 19, 30 produced a reduction in LOG of monophasic $S$. Typhimurium higher than the positive control $L$. reuteri Protectis ${ }^{\circledR}$ and LAB 8, $18,36 \mathrm{had}$ an increase growth in MRS also higher than the positive control (Table 2). Wilcoxon test showed that strain
18 had the greatest value of inhibition, which was both higher than the positive control and significantly higher than the other six selected LAB from spot-on-lawn $(p<0.05)$ and strain 8 the greatest value of proliferation $(p<0.05)$.

Based on these results, LAB 18 was selected as a possible probiotic candidate due to its capacity to inhibit significantly monophasic $S$. Typhimurium in XLD (Fig. 4) and to grow even more in presence of monophasic $S$. Typhimurium in MRS (Fig. 5). 


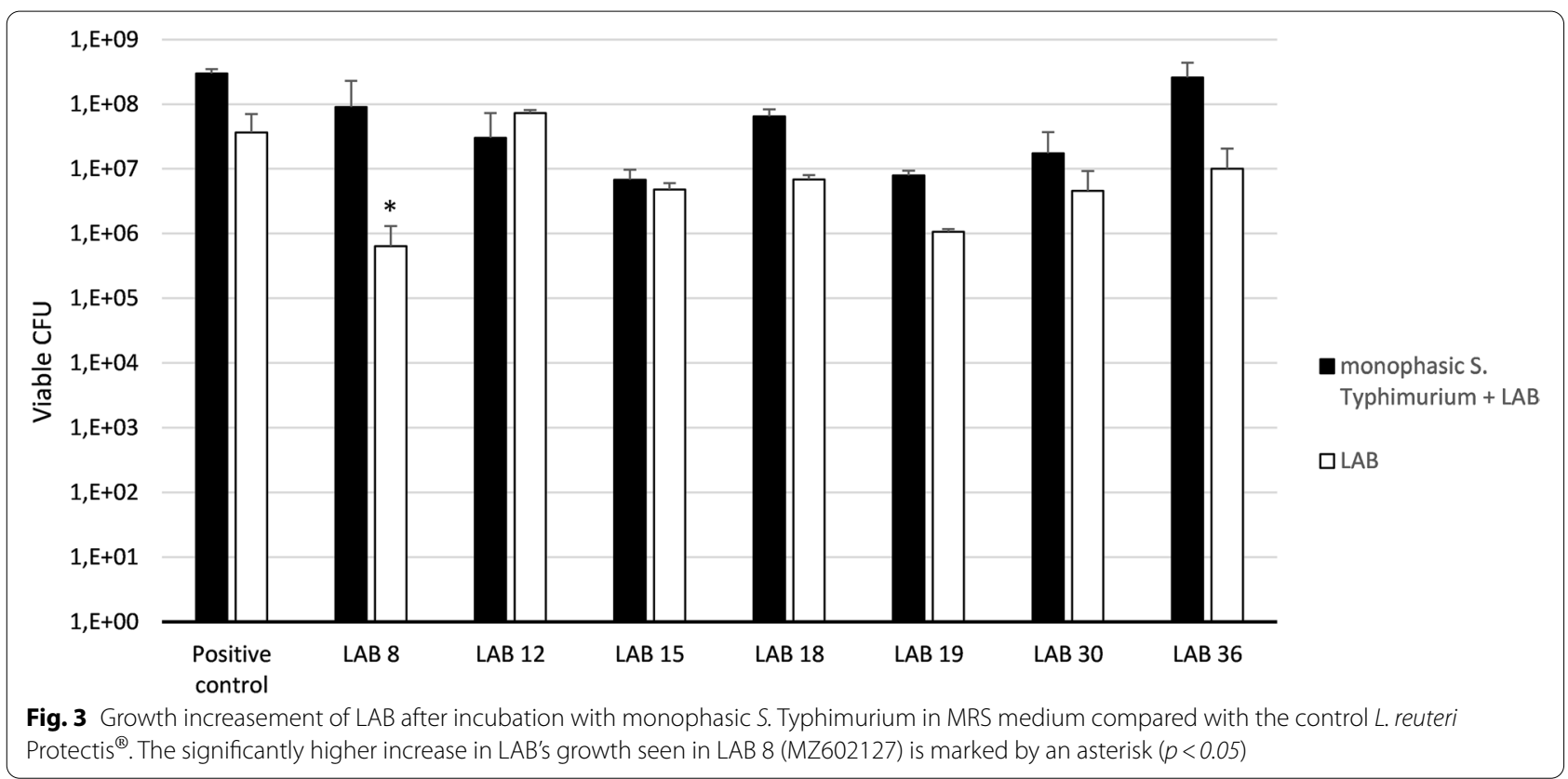

Table 2 Values of inhibition of monophasic S. Typhimurium by $\angle A B$ in XLD medium, and increased growth of $L A B$ after incubation with monophasic $S$. Typhimurium in MRS medium

\begin{tabular}{lll}
\hline LAB & $\begin{array}{l}\text { reduction in LOG } \\
\text { monophasic } S . \\
\text { Typhimurium (XLD) }\end{array}$ & $\begin{array}{l}\text { increase in LOG } \\
\text { LAB } \\
\text { (MRS) }\end{array}$ \\
\hline $\begin{array}{l}\text { Positive control } L .^{\text {reuteri Protectis }}{ }^{\circledR} \\
\text { ren }\end{array}$ & $\mathbf{1 . 7 4}$ & $\mathbf{0 . 9 1}$ \\
$\mathbf{8}$ & 0.68 & $\mathbf{2 . 1 6 ^ { * }}$ \\
12 & 1.55 & -0.39 \\
15 & 1.62 & 0.15 \\
$\mathbf{1 8}$ & $\mathbf{2 . 0 0 ^ { * }}$ & $\mathbf{0 . 9 8}$ \\
$\mathbf{1 9}$ & $\mathbf{1 . 7 5}$ & 0.87 \\
$\mathbf{3 0}$ & $\mathbf{1 . 7 9}$ & 0.58 \\
$\mathbf{3 6}$ & 0.25 & $\mathbf{1 . 4 1}$ \\
\hline
\end{tabular}

(*) Significantly higher values in LAB 8 and 18 ( $p<0.05$ in both cases)

\section{Antibiotic susceptibility profile of selected LAB and monophasic $S$. Typhimurium strain}

Antibiotic susceptibility profiles showed that most of LAB were sensitive to all antibiotics tested. Values demonstrated that the strains were sensitive to betalactam antibiotics such as ampicillin, to aminoglycosides such as gentamicin and to other broad-spectrum antibiotics such as chloramphenicol. Only strains 12 , 15,30 and 36 were resistant to some of them and the monophasic $S$. Typhimurium strain used was resistant to ampicillin (Table 3).
Survival after exposition to different conditions

All the LAB selected from the co-culture method grew in MRS broth at 25,30 and $40^{\circ} \mathrm{C}$, under anaerobiosis. They were also able to grow after conventional freeze-drying and after storage at -20 and $-80^{\circ} \mathrm{C}$ for 30 days. Under such conditions, the bacterial viability after incubation was $89.9 \%$ of the one found in the control cultures (MRS broth, $37^{\circ} \mathrm{C}$, anaerobiosis).

\section{Molecular characterization}

Strains that showed better results than the commercial positive control L. reuteri Protectis ${ }^{\circledR}$ in the co-culture assay (LAB 8, LAB 18, LAB 19, LAB 30 and LAB 36) (Table 2) were identified by molecular characterization, corrected and aligned by ClustalW with MEGA7 (Supplemental file). Based on the sequence obtained, LAB 8 , LAB 18, LAB 19, LAB 30 and LAB 36, all obtained from Salmonella-negative dogs, were identified as Ligilactobacillus animalis (MZ602127), Ligilactobacillus salivarius (MZ602128) (basionym: Lactobacillus salivarius), Ligilactobacillus salivarius (MZ602129), Lactobacillus reuteri (MZ602130) and Ligilactobacillus salivarius (MZ602131), respectively (Table 4).

In addition, LAB isolated from Salmonella-positive dogs were also sequenced. LAB identified from Salmonella-positive dogs were Enterococcus faecium (3), Enterococcus faecalis (3), Enterococcus gilvus (2), Limosilactobacillus ingluviei (2) and Lactobacillus johnsonii (1), Lactobacillus crispatus (1) (Table 4). 


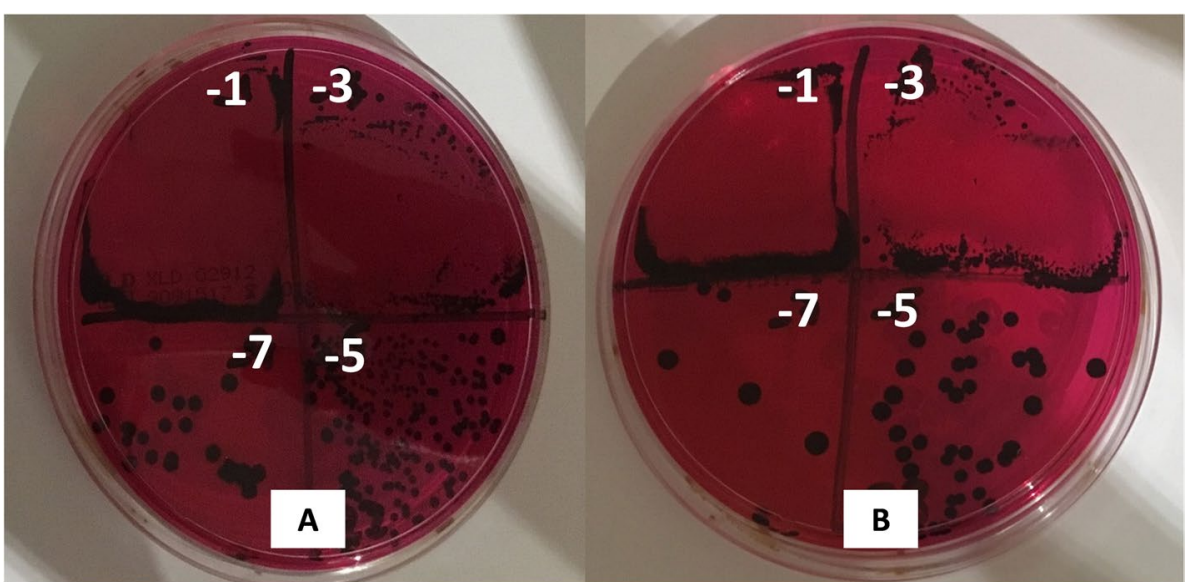

Fig. 4 Monophasic S. Typhimurium growth in XLD. A monophasic S. Typhimurium control B monophasic S. Typhimurium co-incubated with LAB 18 (MZ602128)

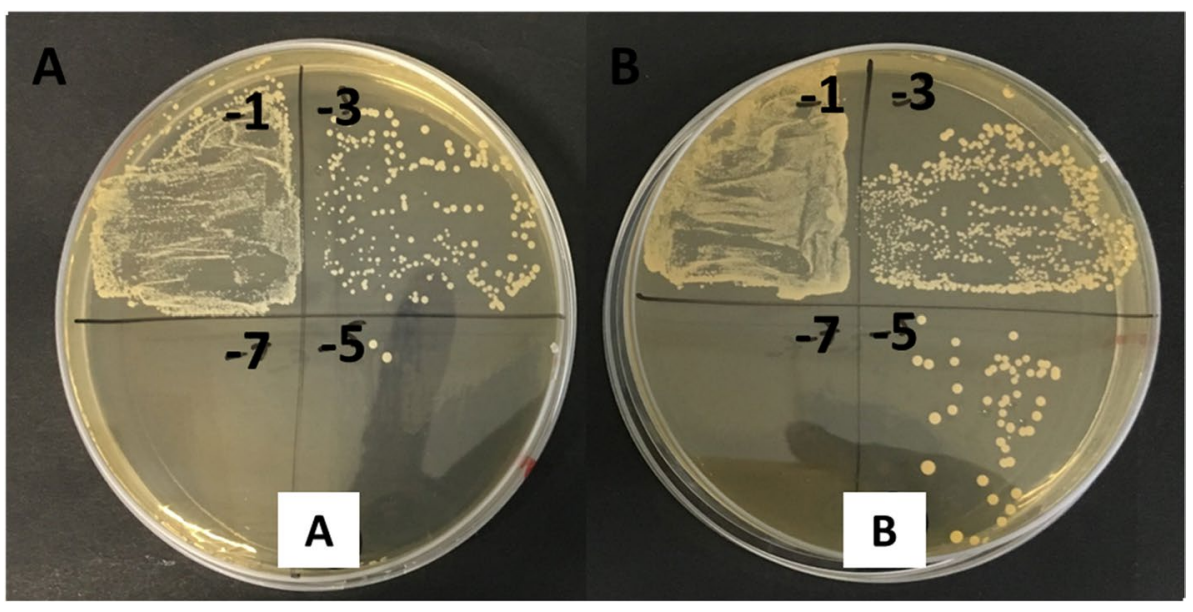

Fig. 5 LAB 18 (MZ602128) growth in MRS. A monoculture of LAB 18 (MZ602128) B LAB 18 (MZ602128) after the co-incubation with monophasic S. Typhimurium

Table 3 Antimicrobial resistance of selected LAB from spot-on-lawn

\begin{tabular}{|c|c|c|c|c|c|c|c|c|c|c|c|}
\hline \multirow[t]{2}{*}{$\mathrm{LAB} / \mathrm{AB}$} & \multicolumn{11}{|c|}{ Antimicrobial susceptibility } \\
\hline & AMP10 & СТХ30 & CAZ30 & GM10 & ND30 & CIP5 & AZM15 & TGC15 & SXT25 & CT10 & C5 \\
\hline 8 & $\mathrm{~S}$ & $S$ & $\mathrm{~S}$ & $\mathrm{~S}$ & $\mathrm{~S}$ & $\mathrm{~S}$ & S & $\mathrm{S}$ & S & $S$ & S \\
\hline 12 & S & $\mathrm{S}$ & S & S & S & $\mathrm{R}$ & S & $\mathrm{S}$ & $S$ & S & $S$ \\
\hline 15 & R & $\mathrm{S}$ & S & S & s & R & S & $\mathrm{S}$ & S & s & S \\
\hline 18 & S & $\mathrm{S}$ & S & $\mathrm{S}$ & s & S & $S$ & S & S & s & S \\
\hline 19 & S & S & S & S & S & S & S & $S$ & $S$ & $S$ & S \\
\hline 30 & $S$ & $S$ & $S$ & $S$ & $S$ & $\mathrm{R}$ & $S$ & $S$ & $S$ & $S$ & S \\
\hline 36 & $S$ & $S$ & $S$ & $S$ & $S$ & R & $S$ & $S$ & $S$ & $S$ & $S$ \\
\hline $\begin{array}{l}\text { Monophasic } \\
\text { S. Typhimurium }\end{array}$ & $\mathrm{R}$ & $S$ & $S$ & $S$ & $S$ & $S$ & $S$ & $S$ & $S$ & $S$ & $S$ \\
\hline
\end{tabular}

AMP Ampicillin, CTX Cefotaxime, CAZ Ceftazidime, GM Gentamicin, ND Nalidixic acid, CIP Ciprofloxacin, AZM Azithromycin, TGC Tigecycline, SXT Trimethoprim sulfamethoxazole, CT Colistin, CChloramphenicol 
Table 4 GenBank accession number(s) for nucleotide sequence

\begin{tabular}{|c|c|c|}
\hline $\begin{array}{l}\text { Bacteria Internal } \\
\text { code }\end{array}$ & Submission number & $\begin{array}{l}\text { Accesion } \\
\text { number to } \\
\text { GenBank }\end{array}$ \\
\hline LAB 8 & SUB10053349 & MZ602127 \\
\hline LAB 18 & SUB10053349 & MZ602128 \\
\hline LAB 19 & SUB10053349 & MZ602129 \\
\hline LAB 30 & SUB10053349 & MZ602130 \\
\hline LAB 36 & SUB10053349 & MZ602131 \\
\hline Dog 1 & SUB10053349 & MZ602132 \\
\hline Dog 2 & SUB10053349 & MZ602133 \\
\hline Dog 3 & SUB10053349 & MZ602134 \\
\hline Dog 4 & SUB10053349 & MZ602135 \\
\hline Dog 5 & SUB10053349 & MZ602136 \\
\hline Dog 6 & SUB10053349 & MZ602137 \\
\hline Dog 7 & SUB10053349 & MZ602138 \\
\hline Dog 8 & SUB10053349 & MZ602139 \\
\hline Dog 9 & SUB10053349 & MZ602140 \\
\hline Dog 10 & SUB10053349 & MZ602141 \\
\hline Dog 11 & SUB10053349 & MZ602142 \\
\hline $\operatorname{Dog} 12$ & SUB10053349 & MZ602143 \\
\hline
\end{tabular}

\section{Antimicrobial activities of the cell free supernatant}

LAB 18 (MZ602128), identified as Ligilactobacillus salivarius was used for the study of cell free supernatant. Results showed that different concentrations of supernatant containing an active compound, were able to inhibit monophasic S. Typhimurium in Mueller Hinton Plate Agar. A dose-dependent response was clearly observed (Fig. 6).

\section{Discussion}

Nowadays, Salmonella is considered one of the four key global causes of diarrheal diseases and it is currently one of the most widely studied bacterial pathogen affecting both domestic and wild animals and humans. Several potential GI pathogens are recognized in dogs, including Salmonella spp. [24]. A previous study [14] has suggested that intestinal LAB macroscopic differences observed in Salmonella-negative dogs and Salmonella-positive dogs could be protecting dogs against Salmonella spp. LAB are the most significant groups of probiotic organisms and probiotic supplementations have been successfully used in the prevention and treatment of acute gastroenteritis [25], and their presence could protect against pathogens infections in humans and animals [26, 27]. Different studies have demonstrated the in vitro and in vivo antimicrobial activities of different LAB in dogs [23] or have studied the effects of selected probiotic strains or probiotic mixtures on the microbiome. However, to our knowledge, no previous works have been carried out evaluating the inhibition activity of GI LAB isolated from Salmonella-negative dogs against this pathogen and have not identified these LAB strains in Salmonella-positive dogs. Based on these considerations, the present work has focused on the screening and selection of the best LAB from Salmonella-negative dogs able to inhibit a monophasic $S$. Typhimurium strain isolated in our previous work [14] from a Salmonella-positive dog. Firstly, a screening of thirty-seven GI LAB isolated from Salmonella-negative dogs were tested in vitro with the spot-on-lawn method. Seven LAB showed an inhibition diameter against monophasic $S$. Typhimurium higher than $2.5 \mathrm{~cm}$ and were tested in a co-culture assay in liquid media using commercial L. reuteri Protectis ${ }^{\circledR}$ as positive control. In this co-culture study, three LAB $(18,19$ and 30) demonstrated the capacity to inhibit monophasic $S$. Typhimurium even more than the commercial control L. reuteri Protectis ${ }^{\circledR}$. These strains were identified by molecular techniques as Ligilactobacillus salivarius (L.salivarius) in all three cases and LAB 18 showed, significantly, the higher logarithmic reduction in monophasic S. Typhimurium, which was both higher than control and significantly higher than the other six selected LAB from spot-on-lawn $(p<0.05)$. Secondly, in the co-culture study, we analyzed the capacity of the seven selected LAB from the spot-on-lawn assay, to grow more in MRS incubated with monophasic $S$. Typhimurium than as monocultures. We found that three strains (LAB 8: MZ602127, LAB 18: MZ602128 and LAB 30: MZ602130) had this capacity compared with the commercial positive control L. reuteri Protectis ${ }^{\circledR}$. LAB 8 (MZ602127) was identified as Ligilactobacillus animalis and LAB 30 (MZ602130) as Limosilactobacillus reuteri. Statistically, L. animalis showed the greatest value of proliferation in comparison with the other six LAB. Our results are in accordance with an in vivo study which has demonstrated that, when $L$. animalis was added, a reduction of enterococci and an increase of lactobacilli counts throughout was observed, indicating that the administration of L. animalis could positively influence composition and metabolism of the intestinal microbiota [28].

Several studies have demonstrated the probiotic attributes of $L$. reuteri and L. animalis in dogs [19, 28-30]. Both strains have been identified in canine milk and their probiotic potential was evaluated through different assays, including survival in conditions that resemble those existing in the GI tract, production of antimicrobial compounds, adherence to intestinal mucin, degradation of mucin and pattern of antibiotic sensitivity [31]. Regarding the digestive tract, $L$. reuteri and $L$. animalis 


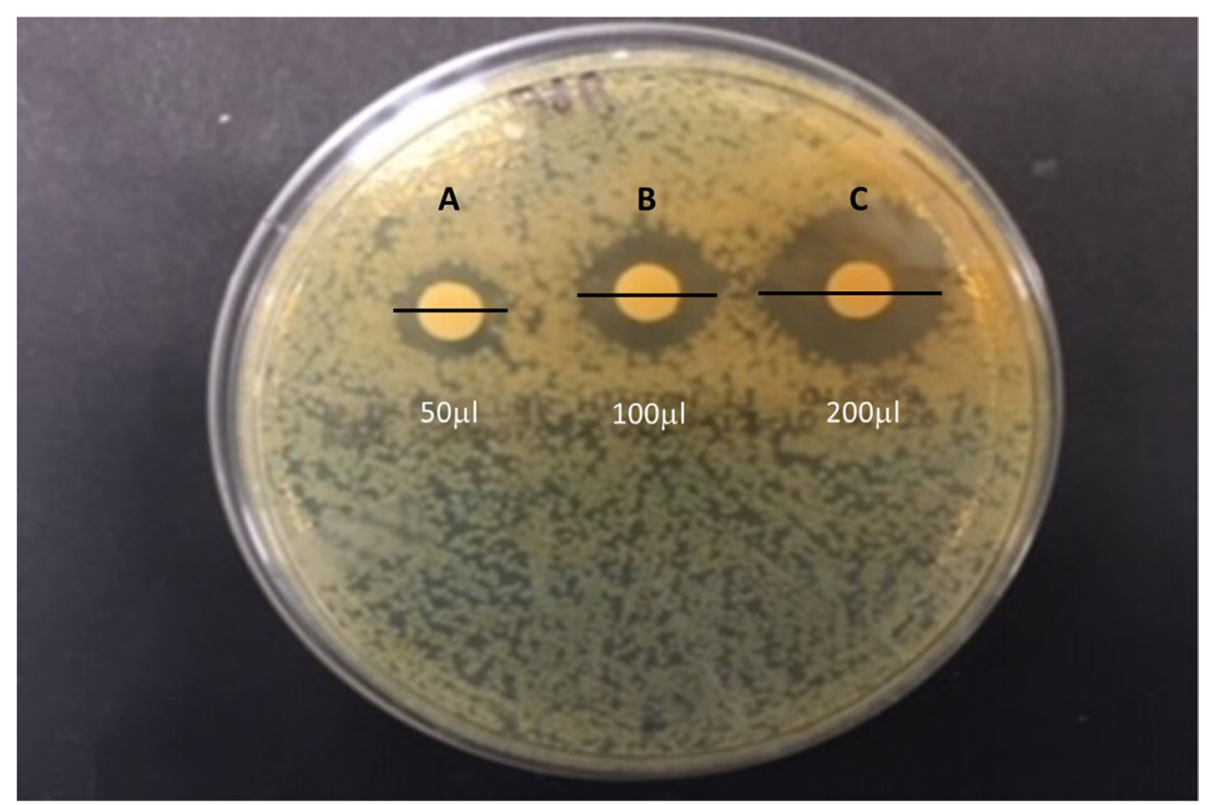

Fig. 6 Agar plate showing the inhibitory activity of filtered supernatant of $L$. salivarius (LAB 18) isolated from Salmonella-negative dogs against monophasic S. Typhimurium: A $50 \mu \mathrm{L}$ of $L$. salivarius cell free supernatant, B $100 \mu \mathrm{L}$ of L. salivarius cell free supernatant and C $200 \mu \mathrm{L}$ of $L$. salivarius cell free supernatant

inhabit commonly all parts of the dog intestine together with L. salivarius and other Lactobacillus species [32].

Considering that LAB 18 (MZ602128) produces, significantly, the higher logarithmic reduction in monophasic $S$. Typhimurium's growth in XLD and had a great value of proliferation in MRS, producing an increase in LOG higher than the positive control, and has been identified in three of the five LAB studied in Salmonella-negative dogs, this strain was selected as the candidate as probiotic for dogs.

Several studies have demonstrated that L. salivarius is part of the canine intestine health [33] and is one of the probiotics available as over-the-counter supplements for dogs [34]. Together with other Lactobacillus species, L. salivarius is able to modify the dominant indigenous jejunal LAB microbiota [35]. Regarding the antimicrobial capacity of L.salivarius, [33] reported that L. salivarius, isolated from Border Collie and German Shorthaired Pointer, showed antimicrobial activity towards Micrococcus luteus.

Studies made in other animal species have shown that $L$. salivarius from bottlenose dolphin can inhibit the growth of $S$. Enteriditis strains isolated from both marine animals and humans [36]. In piglets, L. salivarius strains have been isolated and its antimicrobial effect has been studied against Salmonella [37, 38]. Also, it has been found that $L$. salivarius has an antimicrobial activity against E. coli and Klebsiella in chicken and prevents
S. Enteriditis colonization [39, 40]. Also, other studies showed that $L$. salivarius could eliminate $S$. Enteriditis from chickens after an oral challenge of the pathogen on day 1 [40]. L. salivarius was also proposed as a probiotic product for administration during the feeding of calves for its capacity of aggregation and its good yield [41]. Moreover, it was seen in cattle that $L$. salivarius exhibits remarkable anti-salmonella activities with total inhibition of Salmonella spp. after $18 \mathrm{~h}$ of co-incubation [42].

To our knowledge, in dogs, no study has demonstrated the effect of $L$. salivarius against Salmonella. O'Mahony et al. [43], revealed that some LAB, specifically Bifidobacterium animalis AHC7, has significant potential for improving canine gastrointestinal health but no $L$. salivarius was identified as a potential probiotic.

Regarding probiotics attributes, $L$. salivarius has been extensively studied [44] as well as $L$. reuteri and $L$. animalis. These LAB are included in the list of taxonomic units proposed for qualified presumption of safety (QPS) status from EFSA and detailed toxicological study was performed following the $\mathrm{FAO} / \mathrm{WHO}$ recommendations [45]. In addition to its safety and functionality, a probiotic candidate must survive in GI conditions and remains several years in the GI tract of dogs [46].

Taking into account that all LAB from Salmonellanegative dogs were identified as $L$. salivarius, $L$. reuteri or $L$. animalis, it is reasonable to think that these strains 
identified in Salmonella-negative dogs may be protecting animals against Salmonella colonization and infection.

In Salmonella-positive dogs, most of the LAB identified belonged to the Enterococcus genus. Rinkinen et al. [47] demonstrated that two strains of E. faecium significantly enhanced the adhesion of Campylobacter jejuni, up to 134.6 and $205.5 \%$. This suggest that E. faecium may thus favor the adhesion and colonization of $C$. jejuni in the dog's intestine, making it a potential carrier and possibly a source of infection [47]. Another study has demonstrated that the supplementation of E. faecium in 12 healthy dogs, kept in households during 18-days, reduces the counts of Clostridium spp. while increasing the counts of Salmonella spp. and Campylobacter spp. in the majority of dogs [48]. In another work published by Rinkinen et al. [47], the ability of certain LAB to inhibit the adhesion of selected canine and zoonotic pathogens was evaluated and they observed that the adhesion of $S$. Typhimurium was not significantly affected by any of the LAB tested; however, L. reuteri, L. animalis and L. salivarius reduced the counts of monophasic $S$. Typhimurium as previously reported.

One of the main arguments for the use of probiotics in preventing and combating digestive disorders in animals is the inhibition of potential pathogenic bacteria by producing a variety of inhibitory substances [26, 49]. In that sense, to identify the active substance from LAB 18, able to inhibit monophasic $S$. Typhimurium, a preliminary study was carried out in our work. Results showed a clear monophasic $S$. Typhimurium inhibition when different supernatant concentrations were employed. To the best of our knowledge, four reports mention bacteriocin production in L. salivarius. Arihara et al. [49] isolated from Japanese grass leaves bacteriocins from $L$. salivarius and Ocaña et al. [50] worked with human vaginal sample bacteriocins. Robredo et al. [51] detected a bacteriocin activity in $61 \%$ of the isolates recovered from fecal samples of 20 pigs, and all bacteriocin-producing L. salivarius isolates strongly inhibited the growth of Staphylococcus aureus and Staphylococcus epidermidis, but none of these bacteriocin-producing L. salivarius isolates showed growth inhibition activity against Enterococcus spp., Bacillus spp., or E. coli and they were not tested towards $S$. Typhimurium. As far as we know, few studies have identified the inhibitory substances, produced by selected L. salivarius strains, able to inhibit $S$. Typhimurium [52].

\section{Conclusions}

We have identified a potential antimicrobial LAB against monophasic S. Typhimurium from Salmonella-negative dogs, not isolated in Salmonella-positive dogs. This LAB, identified as Ligilactobacillus salivarius could prevent monophasic $S$. Typhimurium colonization, protecting dogs against Salmonella infection or inhibiting its activity if the infection occurs. Further in vitro and in vivo studies must be carried out to evaluate the probiotic attributes and to identify the active compound responsible of the inhibition.

\section{Material and methods}

\section{Obtention of LAB isolates}

Obtention of LAB from Salmonella-positive dogs and Salmonella-negative dogs was carried out as previously described [14]. As it was indicated in the previous work, Salmonella-positive dogs were animals where Salmonella spp. were isolated from their faeces while in Salmonellanegative dogs no Salmonella spp. were found. All animals were handled according to the principles of animal care published by Spanish Royal Decree 53/2013 [53]. Sampled collection was approved by the Ethics Committee and Animal Experimentation of UCH-CEU University.

Samples were inoculated in the MRS agar and incubated $24-48 \mathrm{~h}$ in anaerobic conditions at $37^{\circ} \mathrm{C}$. After incubation, morphologically different colonies obtained from Salmonella-positive dogs and Salmonella-negative dogs were tested as Gram-positive bacteria using the Gram staining method and as catalase positive and were frozen at $-80^{\circ} \mathrm{C}$ and stored until their use.

Moreover, data from each dog were also collected by a questionnaire that included data related with the environment: where animals were housed, breed, age, gender, diet or type of food, contact with other animal species and the source of water. All questionnaires were completed and submitted together with the samples to the laboratory.

\section{Inhibition assays in solid and liquid media: spot-on-lawn and co-culture methods}

Monophasic $S$. Typhimurium strain used for inhibition assays in this study was isolated from dogs' faeces in the same previous work [14] and spot-on-lawn and co-culture assays were performed in three independent experiments. Control medium was carried out in both assays.

\section{Inhibition assays in solid media: spot-on-lawn method}

Thirty-seven LAB from Salmonella-negative dogs were selected for inhibition assays. LAB were tested by the spot-on-lawn method and the technique was adapted from the method described by Harris et al. [54]. For this method, $5 \mathrm{~mL}$ of an overnight LAB culture in liquid MRS were spotted onto the surface of an MRS agar plate. The plates were incubated at $37^{\circ} \mathrm{C}$ in anaerobic conditions $(24 \mathrm{~h})$. After incubation, MRS agar plates were overlaid with $10 \mathrm{~mL}$ of Nutrient soft agar $(0.80 \%$ 
agar) seeded with an overnight broth culture of monophasic $S$. Typhimurium. The overlay was incubated at $37^{\circ} \mathrm{C}$ for $24 \mathrm{~h}$ and then, the plates were examined for zones of inhibition in the monophasic $S$. Typhimurium cell lawn. Inhibition was determined by measuring the diameter of clear zones $(\mathrm{cm})$ around the LAB spots. For inhibition halos control, an antibiotic disk selected by its inhibition activity against monophasic $S$. Typhimurium (ciprofloxacin) was used. All assays were performed in triplicate.

\section{Inhibition assays in liquid media: co-culture method}

For this experiment, only LAB with the highest inhibition result in the spot-on-lawn method (those with more than $2.5 \mathrm{~cm}$ of inhibition halo) were tested. The method was adapted from the previously described by Adetoye et al. [42]. For the co-culture, $10 \mathrm{ml}$ of Todd Hewitt broth (THB) was inoculated with LAB and monophasic $S$. Typhimurium growth at the same $\mathrm{OD}_{600}$ in the stationary phase. Two experimental controls, which consisted of a monoculture of each LAB and a monoculture of monophasic $S$. Typhimurium, were set up. All samples were incubated at $37^{\circ} \mathrm{C}$ for $24 \mathrm{~h}$. To determine the viable counts $(\mathrm{CFU} / \mathrm{mL})$ of both monocultures and the co-cultures, serial ten-fold dilutions were carried out and $25 \mu \mathrm{L}$ of each dilution were plated on both XLD and MRS agar to control the growth of monophasic $S$. Typhimurium and $\mathrm{LAB}$, respectively. The commercial strain Lactobacillus reuteri Protectis ${ }^{\circledR}$ (DSM17938, Casen Recordatori) (L. reuteri Protectis ${ }^{\circledR}$ ) used in humans against Salmonella infection was used as positive control. All assays were performed in triplicate and the log transformation was applied to the average.

\section{Antibiotic susceptibility profile of selected LAB}

The antibiotic susceptibility profile of the LAB isolates was conducted using the agar disk diffusion method established by the Clinical and Laboratory Standards Institute [55]. Antimicrobial agents and concentrations used in this study were those set forth in Decision 2013/653 (European Union 2013:653): ampicillin (10 $\mu \mathrm{g})$, cefotaxime $(30 \mu \mathrm{g})$, ceftazidime $(30 \mu \mathrm{g})$, gentamicin $(10 \mu \mathrm{g})$, nalidixic acid $(30 \mu \mathrm{g})$, ciprofloxacin $(5 \mu \mathrm{g})$, azithromycin $(15 \mu \mathrm{g})$, tigecycline $(15 \mu \mathrm{g})$, trimethoprimsulfamethoxazole $(25 \mu \mathrm{g})$, colistin $(10 \mu \mathrm{g})$ and chloramphenicol $(5 \mu \mathrm{g})$. Antimicrobial susceptibility was tested according to the European Committee on Antimicrobial Susceptibility Testing (EUCAST) guidelines [56]. The source for zone diameters used for interpretation of the test was https://www.eucast.org/clinical_breakpoints/ . Zone diameters were interpreted and categorized as susceptible, intermediate or resistant according to the EUCAST clinical breakpoint tables.

\section{Survival after exposition to different conditions}

The survival of seven LAB, selected from the spot-onlawn method, after exposure to different conditions for probiotic applications, was tested in MRS broth. The following growth conditions were assayed under anaerobiosis: 25,30 and $40^{\circ} \mathrm{C}$, freeze-drying and storage at $-20^{\circ} \mathrm{C}$ and $-80^{\circ} \mathrm{C}$ for 30 days in the presence of $15 \%$ (v/v) glycerol. All assays were performed in triplicate. MRS cultures incubated at $37^{\circ} \mathrm{C}$ under anaerobiosis were used as controls.

\section{Molecular characterization}

Selected LAB, isolated from Salmonella-negative dogs, from the co-culture assay, were used for molecular characterization. Also, LAB isolated from Salmonellapositives dogs, were selected for molecular characterization to compare the sequence between positive and negative dogs. LAB were processed for genomic DNA extraction and identified based on PCR amplification and sequencing of $16 \mathrm{~S}$ rRNA gene using bacterial universal primers (27F 5'- AGAGTTTGATCC TGGCTCAG and 1492R 5'-GGTT ACCTTGTTA CGACTT) [23]. The PCR was performed in $25 \mu \mathrm{l}$ reaction volumes containing $2 \mathrm{X}$ Taq Master Mix, $0.25 \mathrm{mM}$ forward primer, $0.25 \mathrm{mM}$ reverse primer and $0.4 \mathrm{ng}$ of genomic DNA and nuclease-free water to make volume $25 \mu \mathrm{l}$. Temperature cycling conditions for PCR were as follows: an initial heating of $95^{\circ} \mathrm{C}$ for $3 \mathrm{~min}$, followed by 40 cycles of denaturation at $95^{\circ} \mathrm{C}$ for $30 \mathrm{~s}$, annealing at $55^{\circ} \mathrm{C}$ for $30 \mathrm{~s}$, extension at $72^{\circ} \mathrm{C}$ for $90 \mathrm{~s}$ and terminating with a $5 \mathrm{~min}$ final incubation of $72^{\circ} \mathrm{C}$. The PCR products were examined with electrophoresis on a $1.5 \%$ w/v agarose gel, stained by Safe Lab nucleic acid stain. The PCR products were purified, and sequenced and analysed for sequence homology by BLAST (http:// www.ncbi.nlm.nih. gov/). The sequences were corrected and aligned by ClustalW with MEGA7: Molecular Evolutionary Genetics Analysis version 7.0 for bigger datasets [23]. Bacterial identification was carried out by comparing the problem sequence with the GenBank database through the Blast application. Only identification with a Query Cover equal to or greater than $98 \%$ and with an E value of 0.0 were considered.

\section{Antimicrobial activities of the cell free supernatant}

For this assay, the higher inhibitory LAB able to significantly inhibit monophasic $S$. Typhimurium in the coculture method was used. A volume of $20 \mathrm{ml}$ culture growing in MRS broth at $37^{\circ} \mathrm{C}$ was obtained and centrifugated at $12,000 \mathrm{~g}$ for $10 \mathrm{~min}$ at $4{ }^{\circ} \mathrm{C}$. Supernatant was neutralized to pH6.5 with $\mathrm{NaOH}$ and filter-sterilised through $0.22 \mu \mathrm{m}$ pore size filters (Millipore). The bacteriocinogenic activity of the cell-free supernatant was 
determined in Mueller-Hinton plate agar disk diffusion. The method was adapted from the previously described by Lee et al. [57]. Monophasic $S$. Typhimurium was inoculated on the surface and $50 \mu$ of LAB supernatant was spotted onto the previously inoculated plate. To identify a dose-response effect, different volumes of the supernatant $(50,100$ and $200 \mu \mathrm{l})$ were concentrated to $50 \mu \mathrm{l}$ and were impregnated in a blank disk and placed also in the Mueller-Hinton agar plate and the diameter of inhibition was quantified. In all these expermients, filtered supernatant was obtained the same day as bacterial growth was made.

\section{Statistical analyses}

Compliance with the assumption of normality was checked for the diameter of the inhibition halos of the initial thirty-seven LAB isolates by means of the Shapiro-Wilk test. The results showed that the variable did not distribute normally. Accordingly, the nonparametric Wilcoxon one-sample signed rank test (SPSS/ PASW Statistics for Windows, Version 18.0. SPSS Inc., Chicago, IL) was used to show the significant differences between the median of the diameters of the inhibition halos and each reference value. The nonparametric Wilcoxon onesample signed rank test (SPSS/ PASW Statistics for Windows, Version 18.0. SPSS Inc., Chicago, IL) was also used for co-culture method. An $\alpha$ level of .05 was considered significant for all analyses.

\section{Supplementary Information}

The online version contains supplementary material available at https://doi. org/10.1186/s12917-021-03070-x.

\section{Additional file 1.}

Additional file 2.

\section{Acknowledgements}

We would like to thank the animal shelters and dog owners for their cooperation during sample collection.

\section{Authors' contributions \\ EJT and EB contributed to the study design, laboratory investigation, data analysis and preparation of the draft manuscript. MT, MB, JQ and AGM pro- vided technical assistance during laboratory investigation. AGM carried out sequences analysis. All authors read and approved the final manuscript.}

\section{Funding}

This work was funded and approved by the precompetitive projects CEU - Banco Santander (FUSP-BS-PPC15/2016), IDOC 18-02 and the grant for carrying out research, development and innovation projects by emerging research groups 2020 (GVA/2020/026), Generalitat Valenciana (Spain). Also, by the Consolidation Aid Program of Indicators CEU-UCH (INDI21/22) and the project from Spanish Ministry of Science and Innovation (Project reference PID2020-119462RA-I00/AEI/10.13039/501100011033).

J. J. Quereda is supported by a "Ramón y Cajal" contract of the Spanish Ministry of Science, Innovation and Universities (RYC-2018-024985-I)".

M. Toquet is supported by a research grant from the CEU-UCH.
Availability of data and materials

The datasets analyzed during this study are available from the corresponding author on reasonable request.

The datasets generated during the current study are available in the GenBank repository, GenBank accession numbers for nucleotide sequences:

SUB10053349 LAB8 MZ602127

SUB10053349 LAB18 MZ602128

SUB10053349 LAB19 MZ602129

SUB10053349 LAB30 MZ602130

SUB10053349 LAB36 MZ602131

SUB10053349 dog1 MZ602132

SUB10053349 dog2 MZ602133

SUB10053349 dog3 MZ602134

SUB10053349 dog4 MZ602135

SUB10053349 dog5 MZ602136

SUB10053349 dog6 MZ602137

SUB10053349 dog7 MZ602138

SUB10053349 dog8 MZ602139

SUB10053349 dog9 MZ602140

SUB10053349 dog10 MZ602141

SUB10053349 dog11 MZ602142

SUB10053349 dog12 MZ602143

\section{Declarations}

\section{Ethics approval and consent to participate}

All animals were handled according to the principles of animal care published by Spanish Royal Decree 53/2013 (Official Spanish State Gazette [BOE], 2013). Sampled collection was approved by the Ethics Committee and Animal Experimentation of UCH-CEU University. Informed oral consent, also approved by the Ethics Committee and Animal Experimentation of the UCH-CEU University, was obtained from the dog owners and shelters at the time of sample collection. Consent was documented in the questionnaire that owners and shelters also completed at the time of sample collection.

\section{Consent for publication}

Not applicable.

\section{Competing interests}

The authors declare that they have no competing interests.

\section{Author details}

${ }^{1}$ Microbiological Agents Associated with Animal Reproduction (ProVaginBIO) Research Group, Departamento Producción y Sanidad Animal, Salud Pública Veterinaria y Ciencia y Tecnología de los Alimentos, Facultad de Veterinaria, Universidad Cardenal Herrera-CEU, CEU Universities, Carrer Tirant lo Blanc, 7 , 46115 Alfara del Patriarca, Valencia, Spain. ${ }^{2}$ Research Group Intracellular Pathogens: Biology and Infection, Departamento Producción y Sanidad Animal, Salud Pública Veterinaria y Ciencia y Tecnología de los Alimentos, Facultad de Veterinaria, Universidad Cardenal Herrera-CEU, CEU Universities, Valencia, Spain.

Received: 13 July 2021 Accepted: 30 October 2021

Published online: 03 January 2022

\section{References}

1. Guardabassi L, Schwarz S, Lloyd DH. Pet animals as reservoirs of antimicrobial-resistant bacteria. J Antimicrob Chemother. 2004;54:321-32.

2. Saputra S, Jordan D, Worthing KA, Norris JM, Wong HS, Abraham R, et al. Antimicrobial resistance in coagulase-positive staphylococci isolated from companion animals in Australia: A one year study. PLoS One. 2017:12:e0176379.

3. Abraham S, O'Dea M, Trott DJ, Abraham RJ, Hughes D, Pang S, et al. Isolation and plasmid characterization of carbapenemase (IMP-4) producing Salmonella enterica Typhimurium from cats. Sci Rep. 2016;6:35527.

4. Platell JL, Cobbold RN, Johnson JR, Heisig A, Heisig P, Clabots C, et al. Commonality among fluoroquinolone-resistant sequence type ST131 
extraintestinal Escherichia coli isolates from humans and companion animals in Australia. Antimicrob Agents Chemother. 2011;55:3782-7.

5. Beck KM, Waisglass SE, Dick HLN, Weese JS. Prevalence of meticillin-resistant staphylococcus pseudintermedius (MRSP) from skin and carriage sites of dogs after treatment of their meticillin-resistant or meticillin-sensitive staphylococcal pyoderma. Vet Dermatol. 2012;23(369-75):e66-7.

6. Klevens RM, Edwards JR, Richards CL, Horan TC, Gaynes RP, Pollock DA et al. Estimating health care-associated infections and deaths in U.S. hospitals, 2002. Public Health Rep. 2007;122:160-6.

7. Carmeli Y. Strategies for managing today's infections. Clin Microbiol Infect. 2008;14:22-31.

8. Anonymous. European surveillance of veterinary antimicrobial consumption (ESVAC). European Medicines Agency 2018. https://www.ema. europa.eu/en/veterinary-regulatory/overview/antimicrobial-resistance/ european-surveillance-veterinary-antimicrobial-consumption-esvac. Accessed 20 May 2021.

9. European Food Safety Authority. The European Union summary report on antimicrobial resistance in zoonotic and indicator bacteria from humans, animals and food in 2017. EFSA J. 2019;17:e05598.

10. Reimschuessel R, Grabenstein M, Guag J, Nemser SM, Song K, Qiu J, et al. Multilaboratory survey to evaluate Salmonella prevalence in diarrheic and nondiarrheic dogs and cats in the United States between 2012 and 2014. J Clin Microbiol. 2017;55:1350-68.

11. Olufemi O, Adetosoye A. Salmonella Typhimurium Typhimurium infection in diarrhoeic and non-diarrhoiec infection in diarrhoeic and non-diarrhoiec dogs in Ibadan, Nigeria. 2009;79. https://www.researchgate.net/ publication/242156294_Salmonella_Typhimurium_Typhimurium_infec tion_in_diarrhoeic_and_non-diarrhoiec_infection_in_diarrhoeic_and_ non-diarrhoiec_dogs_in_lbadan_Nigeria_dogs_in_lbadan_Nigeria.

12. Seepersadsingh $N$, Adesiyun AA, Seebaransingh R. Prevalence and antimicrobial resistance of Salmonella spp. in non-diarrhoeic dogs in Trinidad. J Vet Med B Infect Dis Vet Public Health. 2004;51:337-42.

13. Jajere SM, Onyilokwu SA, Adamu NB, Atsanda NN, Saidu AS, Adamu SG, et al. Prevalence of salmonella infection in dogs in Maiduguri, northeastern Nigeria. Int J Microbiol. 2014;2014:392548.

14. Bataller E, García-Romero E, Llobat L, Lizana V, Jiménez-Trigos E. Dogs as a source of Salmonella spp. in apparently healthy dogs in the Valencia region. Could it be related with intestinal lactic acid bacteria? BMC Vet Res. 2020;16:268.

15. Lowden $P$, Wallis $C$, Gee N, Hilton A. Investigating the prevalence of Salmonella in dogs within the midlands region of the United Kingdom. BMC Vet Res. 2015;11:239.

16. Tsai H-J, Huang H-C, Lin C-M, Lien Y-Y, Chou C-H. Salmonellae and campylobacters in household and stray dogs in northern Taiwan. Vet Res Commun. 2007;31:931-9.

17. Wright JG, Tengelsen LA, Smith KE, Bender JB, Frank RK, Grendon JH, et al. Multidrug-resistant Salmonella Typhimurium in four animal facilities. Emerging Infect Dis. 2005;11:1235-41.

18. Servin AL. Antagonistic activities of lactobacilli and bifidobacteria against microbial pathogens. FEMS Microbiol Rev. 2004;28:405-40.

19. Coman MM, Verdenelli MC, Cecchini C, Belà B, Gramenzi A, Orpianesi C, et al. Probiotic characterization of Lactobacillus isolates from canine faeces. J Appl Microbiol. 2019;126:1245-56.

20. White R, Atherly T, Guard B, Rossi G, Wang C, Mosher C, et al. Randomized, controlled trial evaluating the effect of multi-strain probiotic on the mucosal microbiota in canine idiopathic inflammatory bowel disease. Gut Microbes. 2017;8:451-66.

21. Wolfenden RE, Pumford NR, Morgan MJ, Shivaramaiah S, Wolfenden AD, Pixley CM, et al. Evaluation of selected direct-fed microbial candidates on live performance and Salmonella reduction in commercial Turkey brooding houses. Poult Sci. 2011;90:2627-31.

22. Shivaramaiah S, Pumford NR, Morgan MJ, Wolfenden RE, Wolfenden $A D$, Torres-Rodríguez A, et al. Evaluation of Bacillus species as potential candidates for direct-fed microbials in commercial poultry. Poult Sci. 2011;90:1574-80.

23. Kumar S, Pattanaik AK, Sharma S, Jadhav SE, Dutta N, Kumar A. Probiotic potential of a Lactobacillus bacterium of canine Faecal-origin and its impact on select gut health indices and immune response of dogs. Probiotics Antimicrob Proteins. 2017:9:262-77.

24. Kantere. Enteric pathogens of dogs and cats with public health implications. Am J Anim Vet Sci. 2014;9:84-94.
25. Grześkowiak Ł, Endo A, Beasley S, Salminen S. Microbiota and probiotics in canine and feline welfare. Anaerobe. 2015;34:14-23.

26. Corcionivoschi N, Drinceanu D, Pop IM, Stack D, Stef L, Julean C, et al. The effect of probiotics on animal health. Sci Papers Animal Sci Biotechnol. 2010:43:35-41.

27. Vieco-Saiz N, Belguesmia Y, Raspoet R, Auclair E, Gancel F, Kempf I, et al. Benefits and inputs from lactic acid Bacteria and their Bacteriocins as alternatives to antibiotic growth promoters during food-animal production. Front Microbiol. 2019:10:57.

28. Biagi G, Cipollini I, Pompei A, Zaghini G, Matteuzzi D. Effect of a Lactobacillus animalis strain on composition and metabolism of the intestinal microflora in adult dogs. Vet Microbiol. 2007;124:160-5.

29. Kim E, Kang Y, Bang T, Lee M, Lee S, Choi I, et al. Characterization of Lactobacillus reuteri BCLR-42 and Lactobacillus plantarum BCLP-51 as novel dog probiotics with innate immune enhancing properties. Korean JVet Res. 2016;56:75-84.

30. McCoy S, Gilliland SE. Isolation and characterization of Lactobacillus species having potential for use as probiotic cultures for dogs. J Food Sci. 2007;72:M94-7.

31. Martín R, Olivares M, Pérez $M$, Xaus J, Torre C, Fernández L, et al. Identification and evaluation of the probiotic potential of lactobacilli isolated from canine milk. Vet J. 2010;185:193-8.

32. Tang Y, Manninen TJK, Saris PEJ. Dominance of Lactobacillus acidophilus in the facultative jejunal Lactobacillus microbiota of fistulated beagles. Appl Environ Microbiol. 2012;78:7156-9.

33. Beasley SS, Manninen TJK, Saris PEJ. Lactic acid bacteria isolated from canine faeces. J Appl Microbiol. 2006:101:131-8.

34. Schmitz S, Suchodolski J. Understanding the canine intestinal microbiota and its modification by pro-, pre- and synbiotics - what is the evidence? Vet Med Sci. 2016;2:71-94.

35. Manninen TJK, Rinkinen ML, Beasley SS, Saris PEJ. Alteration of the canine small-intestinal lactic acid bacterium microbiota by feeding of potential probiotics. Appl Environ Microbiol. 2006;72:6539-43.

36. Diaz MA, Bik EM, Carlin KP, Venn-Watson SK, Jensen ED, Jones SE, et al. Identification of Lactobacillus strains with probiotic features from the bottlenose dolphin (Tursiops truncatus). J Appl Microbiol. 2013;115:1037-51.

37. Casey PG, Casey GD, Gardiner GE, Tangney M, Stanton C, Ross RP, et al. Isolation and characterization of anti-Salmonella lactic acid bacteria from the porcine gastrointestinal tract. Lett Appl Microbiol. 2004;39:431-8.

38. Kim PI, Jung MY, Chang Y-H, Kim S, Kim S-J, Park Y-H. Probiotic properties of Lactobacillus and Bifidobacterium strains isolated from porcine gastrointestinal tract. Appl Microbiol Biotechnol. 2007;74:1103-11.

39. A.C M, Hinton A Jr, H M. Inhibition of Growth of Escherichia coli, Salmonella typhimurium, and Clostridia perfringens on Chicken Feed Media by Lactobacillus salivarius and Lactobacillus plantarum. Int J Poult Sci. 2004;3. https://doi.org/10.3923/ijps.2004.603.607.

40. Pascual M, Hugas M, Badiola JI, Monfort JM, Garriga M. Lactobacillus salivarius CTC2197 prevents Salmonella enteritidis colonization in chickens. Appl Environ Microbiol. 1999;65:4981-6.

41. Frizzo L, Soto LP, Bertozzi E, Sequeira G, Martí LE, Rosmini M. Evaluación in Vitro de las Capacidades Probióticas Microbianas Orientadas al Diseño de Inóculos Probióticos Multiespecie para Ser Utilizados en la Crianza de Terneros. Rev FAVE Cienc Vet. 2006;5:61-72.

42. Adetoye A, Pinloche E, Adeniyi BA, Ayeni FA. Characterization and antisalmonella activities of lactic acid bacteria isolated from cattle faeces. BMC Microbiol. 2018;18:96.

43. O'Mahony D, Murphy S, Boileau T, Park J, O'Brien F, Groeger D, et al. Bifidobacterium animalis $A H C 7$ protects against pathogen-induced NF-KB activation in vivo. BMC Immunol. 2010;11:63.

44. Messaoudi S, Manai M, Kergourlay G, Prévost H, Connil N, Chobert J-M, et al. Lactobacillus salivarius: bacteriocin and probiotic activity. Food Microbiol. 2013;36:296-304.

45. Morelli L, Capurso L. FAO/WHO guidelines on probiotics: 10 years later. J Clin Gastroenterol. 2012;46(Suppl):S1-2.

46. Mitsuoka T, Kimura N, Kobayashi A. Studies on the composition of the fecal flora of healthy dogs with the special references of Lactobacillus flora and Bifidobacterium flora (author's transl). Zentralbl Bakteriol Orig A. 1976;235:485-93.

47. Rinkinen M, Jalava K, Westermarck E, Salminen S, Ouwehand AC. Interaction between probiotic lactic acid bacteria and canine enteric pathogens: 
a risk factor for intestinal Enterococcus faecium colonization? Vet Microbiol. 2003;92:111-9.

48. Vahjen W, Männer K. The effect of a probiotic Enterococcus faecium product in diets of healthy dogs on bacteriological counts of Salmonella spp., Campylobacter spp. and Clostridium spp. in faeces. Arch Tierernahr. 2003;57:229-33.

49. Arihara K, Ogihara S, Mukai T, Itoh M, Kondo Y. Salivacin 140, a novel bacteriocin from Lactobacillus salivarius subsp. salicinius T140 active against pathogenic bacteria. Lett Appl Microbiol. 1996:22:420-4.

50. Ocaña VS, De Ruiz P, Holgado AA, Nader-Macías ME. Characterization of a bacteriocin-like substance produced by a vaginal Lactobacillus salivarius strain. Appl Environ Microbiol. 1999;65:5631-5.

51. Robredo B, Torres C. Bacteriocin production by Lactobacillus salivarius of animal origin. J Clin Microbiol. 2000;38:3908-9.

52. Maldonado NC, de Ruiz CS, Otero MC, Sesma F, Nader-Macías ME. Lactic acid bacteria isolated from young calves--characterization and potential as probiotics. Res Vet Sci. 2012;92:342-9.

53. BOE.es - Documento BOE-A-2013-1337. https://www.boe.es/eli/es/rd/ 2013/02/01/53. Accessed 11 Feb 2021.

54. Harris $L$, Daeschel MA, Stiles ME, Klaenhammer TR. Antimicrobial activity of lactic acid Bacteria against listeria monocytogenes. J Food Prot. 1989:52:384-7.

55. vet01s_sample.pdf. https://clsi.org/media/1530/vet01s_sample.pdf. Accessed 27 Jan 2020

56. EUCAST: Clinical breakpoints and dosing of antibiotics. https://www. eucast.org/clinical_breakpoints/. Accessed 20 May 2021.

57. Lee S-C, Lo H-J, Fung C-P, Lee N, See L-C. Disk diffusion test and E-test with enriched Mueller-Hinton agar for determining susceptibility of Candida species to voriconazole and fluconazole. J Microbiol Immunol Infect. 2009;42:148-53.

\section{Publisher's Note}

Springer Nature remains neutral with regard to jurisdictional claims in pub-

lished maps and institutional affiliations.

- fast, convenient online submission

- thorough peer review by experienced researchers in your field

- rapid publication on acceptance

- support for research data, including large and complex data types

- gold Open Access which fosters wider collaboration and increased citations

- maximum visibility for your research: over 100M website views per year

At BMC, research is always in progress.

Learn more biomedcentral.com/submissions 